\title{
Association between Histone Deacetylase 9 Gene Polymorphism and Stroke in Chinese Han Population
}

\author{
Xitong Yang, ${ }^{1}$ Hongyang Xu, ${ }^{2}$ Dan Liu, ${ }^{1}$ Rong Ma, ${ }^{1}$ Yuanyuan Zhang, ${ }^{1}$ Guangming Wang \\ Genetic Testing Center,' The First Affiliated hospital of Dali University, Dali, China \\ Deparment of Encephalopathy, ${ }^{2}$ Hospital of Traditional Chinese Medicine, Guangde, China
}

Objective : To explore the correlation between the polymorphism of histone deacetylase 9 gene (rs1060499865, rs723296, rs957960) and ischemic stroke (IS) in Chinese Han population in Dali region.

Methods : This study included 155 IS patients and 128 healthy physical examinees. TaqMan-polymerase chain reaction technology and multivariate logistic regression were performed.

Results : In the case group, there was no polymorphism of rs1060499865 observed in the two groups; whereas on the rs723296 locus the frequencies of $C$ allele and TC genotype were significantly higher than that in the control group, alleles $C$ and T were associated with a 2.158-fold increase in IS risk, and genotypes TC and TT were associated with a 2.269-fold increase in IS risk. The locus rs957960 exhibited no significant difference between the two groups.

Conclusion : An association between rs723296 and the risk of IS was found in the Chinese Han population in Dali region. No significant association was found between rs1060499865, rs957960 and IS in the Chinese Han population in Dali region.

Key Words : Stroke · HDAC9 protein · Polymorphism.

\section{INTRODUCTION}

Stroke is a multifactor disease, the interaction between environmental and genetic factors plays a significant role in the pathogenesis of stroke. The prevalence of stroke is on the rise as the aging process of the global population is constantly increasing ${ }^{9,22)}$. About 15 million people suffer stroke worldwide every year, of whom $20 \%$ leads to fatality, more than $50 \%$ lose their ability to take care of themselves and to perform their daily activities ${ }^{30}$. Ischemic stroke (IS) accounts for more than $85 \%$ of strokes, which is increasing every year in China, and shows a trend of younger population suffering from IS $^{28)}$. IS being a complex disease is caused by multiple factors; family and twins' studies have indicated that genetic factors play an important role in the pathogenesis of IS $^{1)}$.

Histone deacetylase 9 (HDAC9) is located on human chro-

- Received : February 12, 2020 •Revised : March 20, 2020 •Accepted : April 14, 2020

- Address for reprints : Yuanyuan Zhang

Deparment of Encephalopathy, The First Affiliated hospital of Dali University, 32 Jiashibo Road, Dali, Yunnan 671000, China

Tel : +86-872-2201096, Fax : +86-872-2201096, E-mail : zhyy@dali.edu.cn, ORCID : https://orcid.org/0000-0002-5263-4895

\section{Guangming Wang}

Deparment of Encephalopathy, The First Affiliated hospital of Dali University, 32 Jiashibo Road, Dali, Yunnan 671000, China

Tel : +86-872-2201096, Fax : +86-872-2201096, E-mail : wgm1991@dali.edu.cn, ORCID : https://orcid.org/0000-0002-0220-1493

This is an Open Access article distributed under the terms of the Creative Commons Attribution Non-Commercial License (http://creativecommons.org/licenses/by-nc/4.0) which permits unrestricted non-commercial use, distribution, and reproduction in any medium, provided the original work is properly cited. 
mosome 7p21, the region associated with neurological diseases and various types of tumors. The HDAC9 gene contains 32 exons with an overall length of about $500 \mathrm{~KB}$. The 3 'end is about $150 \mathrm{~KB}$ away from the downstream TWIST gene, mainly encoding HDAC9 and HDRP enzyme protein subtypes ${ }^{16)}$. HDAC9 is primarily found in the cytoplasm, shuttling between the nucleus and cytoplasm, while regulating acetylation of histones and nonhistones ${ }^{11)}$. HDAC9 acts as an important regulator of histone acetylation to control gene transcription, rendering the gene promoter inaccessible to the transcriptional regulatory elements, thus inhibiting transcription. Disturbances in the balance of nonhistone acetylation and deacetylation affect many aspects of normal cellular functioning. To date, the HDAC9 gene has been identified as the strongest risk locus for large-vessel strokes ${ }^{14)}$. Hypoacetylation at gene promoters usually result in transcriptional inhibition by restraining accessibility of transcriptional machinery to chromatin ${ }^{24)}$. Monocytes, macrophages, T lymphocytes, vascular endothelial, and smooth muscle cells express HDAC9 ${ }^{15)}$. HDAC9 was upregulated in human carotid and aortic atherosclerotic plaques ${ }^{17)}$ and the plasma HDAC9 expression was found to be significantly higher in patients with coronary artery disease ${ }^{25)}$.

Genome-wide association study revealed a new IS susceptibility HDAC9 gene. The loci rs11984041 and rs2107595 of HDAC9 polymorphism were found to significantly increase the risk of atherosclerotic $\mathrm{IS}^{8,21)}$. HDAC9 has been reported to regulate gene expression by modifying the chromatin structure, though DNA sequence remains unchanged. HDAC9 was revealed in whole genome sequencing studies that was found associate closely with IS; the HDAC9 gene was identified as a highly risky gene responsible for IS. HDAC9 zymoprotein is found abundantly in brain, muscles, myocardium, and vascular endothelial cells. The HDAC9 gene has been reported to have a significant role in atherosclerosis, inflammatory response, lipid metabolism, and vascular regeneration ${ }^{23)}$. The expression of $H D A C 9$ was found to be upregulated in the case of human atherosclerotic plaques ${ }^{20)}$.

Presently, few studies have already reported a correlation between HDAC polymorphism and IS; however, this study explores the relationship between the polymorphism of three single nucleotide polymorphisms (SNP) loci in HDAC9 (rs1060499865, rs723296, and rs957960) and stroke patients in Dali region of China. Here TaqMan-polymerase chain reaction (PCR) technology was used to investigate the association between the HDAC9 polymorphism and IS in the Chinese Han population, aiming to supplement data for further exploring the genetic basis of IS.

\section{MATERIALS AND METHODS}

\section{Study subjects}

The study protocol was approved by the Medical Ethics Committee of the First Affiliated Hospital of Dali University (No. 81360206), and signed informed consent was obtained from all the study participants. From December 2017 to August 2018, 155 IS patients were admitted to the neurology department of the First Affiliated Hospital of Dali University. One hundred and twenty-eight healthy subjects from the outpatient department of our hospital were included in this study as the control group. Following inclusion criteria were considered for forming the IS group : 1) conformed diagnosis of IS based on the diagnostic criteria for cerebrovascular diseases provided in the fourth national conference ${ }^{3)}$ and 2) existing IS patients in Dali whose is living there for more than three generations. Exclusion criteria are as follows : 1) subjects with severe diseases such as heart, kidney, liver, bone diseases and cancer as well as serious infections such as acquired immunodeficiency syndrome and syphilis; 2) people with severe neurological deficits; and 3) no consanguinity was reported between the selected healthy subjects and IS patients.

The control group members were free from cardiovascular and cerebrovascular diseases, autoimmune disorders, malignant tumor, immunological disease, neurological deficits, and severe hepatic and renal dysfunction. No sib ship existed between the selected individuals and IS patients, who were hailed from Dali region whose more than three generations have been living there. Certain demographic and clinical data were collected, such as age, sex, fasting blood glucose (FBG), red blood cells (RBC), white blood cells (WBC), cholesterol, triglycerides (TG), low-density lipoprotein (LDL), systolic pressure, diastolic pressure (DBP), and body mass index (BMI).

\section{DNA extraction}

A portion of $5 \mathrm{~mL}$ of venous blood collected from each subject was stored in tubes containing ethylene diaminetetraacetic acid. Sample DNA was extracted using the QIAGEN DNA 
extraction kit. Genomic DNA extract ion was performed according to manufacturer's protocols (Bomaide Technology, Beijing, China), and the extract was stored finally at $-80^{\circ} \mathrm{C}$ until analysis.

\section{Design and synthesis of primer probes}

Based on NCBI database (https://www.ncbi.nlm.nih.gov/ snp/), rs1060499865, rs723296, and rs957960 loci of the gene HDAC9 were selected. The amplification was performed in 25 $\mu \mathrm{L}$ volume, $12.5 \mu \mathrm{L} 2 \times$ Taq enzyme mixture, $0.5 \mu \mathrm{L}$ CF forward primer, $0.5 \mu \mathrm{L}$ TF forward primer, $1 \mu \mathrm{L}$ R reverse primer,

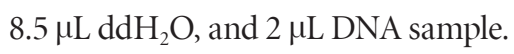

\section{Reaction conditions}

The reaction conditions followed during the experiments are as follows : predegeneration at $95^{\circ} \mathrm{C}$ for 5 minutes, denaturing at $95^{\circ} \mathrm{C}$ for 10 seconds, annealing at $60^{\circ} \mathrm{C}$ for 30 seconds, followed by 30 cycles, extending at $72^{\circ} \mathrm{C}$ for 2 minutes, and conservation at $16^{\circ} \mathrm{C}$ for 5 minutes were followed. Primers and probes were designed using Primer 5 software (Premier, Quebec, Canada). The sequences of primers and probes were procured from Anhui General Biosystems, Inc. (Chuzhou, Anhui, China) and are listed in Table 1.

\section{Statistical analysis}

The statistical analyses were performed using Microsoft Excel (Microsoft, Redmond, WA, USA) and SPSS version 19.0 software (SPSS, IBM, Armonk, NY, USA). Mean \pm standard deviation or standard error values of the continuous variables are presented. Count data were performed using $t$ test; quanti- tative data analyses were performed using the chi-squared test. Univariate and multivariate logistic regression analyses were used to derive the odds ratio (OR). Hardy-Weinberg equilibrium (HWE) was assessed using the SPSS software and Microsoft Excelget corresponding value for determining HDAC9 SNP genotypes distribution in controls. Statistical significance was defined as two-sided $p<0.05$.

\section{RESULTS}

\section{Clinical characteristics}

The IS patients' group comprising 155 patients included 90 males and 65 females, with an average age of $58.63 \pm 12.97$ years. The control group included 128 subjects with 76 males and 50 females, their age on average being $57.59 \pm 14.01$. No significant differences in factors such as age, sex, DBP, and BMI values were recorded between controls and IS but were recorded in the values of FBG, WBC, RBC, TC, TG, LDL, and SBP, and the observed differences are shown in Table 2.

\section{HWE test}

In both the IS and control groups, the locus rs1060499865 exhibited no polymorphism; whereas for rs723296, the $\chi^{2}$ and $p$ values were 0.211 and 0.646 for the control group and 1.19 and 0.27 for the IS group, respectively; and for rs957960, the $\chi^{2}$ and $p$ values were 0.289 and 0.59 for control group and 1.041 and 0.308 for the IS group, respectively. All $p>0.05$ values indicate that the distribution of the data in this study represents the gene distribution of the population.

Table 1. PCR primer sequences used in this study

\begin{tabular}{|c|c|c|c|}
\hline SNP & Polymorphism & Probe name & Sequence \\
\hline \multirow[t]{3}{*}{ rs1060499865 } & $\mathrm{C} / \mathrm{T}$ & CC & 5' -ACGCATGGCTGTTGGCTGTGTCATC-3' \\
\hline & & $\mathrm{TT}$ & 5' -ACGCATGGCTGTTGGCTGTGTCATT-3' \\
\hline & & Reverse & 5'-CTGAGGCCACTTTGGAAGCCAGCTC-3' \\
\hline \multirow[t]{3}{*}{ rs723296 } & $\mathrm{C} / \mathrm{T}$ & $\mathrm{CC}$ & 5' -ACATTTTCTTTTTTGCTTATGCATAC-3' \\
\hline & & $\mathrm{TT}$ & 5'-ACATTTTCTTTTTTGCTTATGCATAT-3' \\
\hline & & Reverse & 5'-GGGAAGGAATTTATGTATGTCAAGT-3' \\
\hline \multirow[t]{3}{*}{ rs957960 } & $\mathrm{A} / \mathrm{C}$ & AA & 5' -TCTTTGAACACAACCATTCCATTA-3' \\
\hline & & CC & 5' -TCTTTGAACACAACCATTCCATTAC-3' \\
\hline & & Reverse & 5'-CACTTAATATCTGCGTTTACATTAT-3' \\
\hline
\end{tabular}

PCR : polymerase chain reaction, SNP : single nucleotide polymorphism 


\section{Comparison of the association of rs1060499865, rs723296, and rs957960 polymorphisms with IS}

No polymorphism in rs1060499865 was observed when a comparison of polymorphism was performed among the three loci rs1060499865, rs723296, and rs957960. The locus rs723296 genotype TC/CC was found to be associated with a

Table 2. Characteristics of IS patients and controls

\begin{tabular}{lccc}
\hline Characteristic & IS patient & Control & $p$-value \\
\hline Age (years) & $58.63 \pm 12.97$ & $57.59 \pm 14.01$ & 0.516 \\
Male gender & $90(58.0)$ & $76(59.3)$ & 0.824 \\
FBG $(\mathrm{mmol} / \mathrm{L})$ & $6.25 \pm 2.73$ & $4.75 \pm 0.87$ & $<0.001$ \\
WBC $\left(10^{12} / \mathrm{L}\right)$ & $7.79 \pm 2.32$ & $6.27 \pm 1.57$ & $<0.001$ \\
RBC $\left(10^{12} / \mathrm{L}\right)$ & $4.81 \pm 0.72$ & $4.97 \pm 0.55$ & 0.004 \\
TC $(\mathrm{mmol} / \mathrm{L})$ & $5.01 \pm 1.29$ & $4.33 \pm 0.42$ & $<0.001$ \\
TG $(\mathrm{mmol} / \mathrm{L})$ & $1.72 \pm 1.40$ & $1.35 \pm 0.75$ & 0.008 \\
LDL $(\mathrm{mmol} / \mathrm{L})$ & $3.05 \pm 1.06$ & $2.82 \pm 0.72$ & 0.033 \\
SBP $(\mathrm{mmHg})$ & $144.92 \pm 25.88$ & $133.85 \pm 15.34$ & $<0.001$ \\
DBP $(\mathrm{mmHg})$ & $86.46 \pm 13.02$ & $84.66 \pm 10.40$ & 0.205 \\
BMI $\left(\mathrm{kg} / \mathrm{m}^{2}\right)$ & $23.15 \pm 3.28$ & $22.84 \pm 3.91$ & 0.478 \\
\hline
\end{tabular}

Values are presented as mean \pm standard deviation or number (\%). IS : ischemic stroke, FBG : fasting blood glucose, WBC : white blood cells, RBC : red blood cells, TC : total cholesterol, TG : triglycerides, LDL : lowdensity lipoprotein, SBP : systolic pressure, DBP : diastolic pressure, BMI : body mass index 2.269-fold increase in IS risk (OR, 2.269; 95\% confidence interval $\left.[\mathrm{CI}], 1.046-4.923 ; \chi^{2}=4.474 ; p=0.034\right)$; allele $\mathrm{C} / \mathrm{T}$ was observed to be associated with a 2.158 -fold increase in IS risk (OR, 2.158; 95\% CI, 1.016-4.582, $\left.\chi^{2}=4.179 ; p=0.041\right)$, indicating that individuals with $\mathrm{C}$ allele and $\mathrm{TC}$ genotype are at a higher risk for IS. The locus rs957960 did not exhibit any significant association between allele $\mathrm{C} / \mathrm{A}$ and genotypes $\mathrm{AC} / \mathrm{AA}$ and CC/AA, and IS, as indicated in Table 3.

\section{DISCUSSION}

Our results indicate that polymorphism of HDAC9 gene rs723296 significantly influences the risk of IS, whereas rs1060499865 and rs957960 did not have any association with IS. Studies have shown that HDAC9 gene stimulates angiogenesis by targeting the microRNA-17-92 group that is antiangiogenic in vascular endothelium ${ }^{10)}$. Therefore, the effect of HDAC9 activity on angiogenesis may be associated with susceptibility to IS.

IS is a complex disease caused as a result of atherosclerosis, which affects the interaction of genomic abnormalities with various environmental factors, and is a major public health burden in China ${ }^{26)}$. Age, diabetes, hypertension, and hypercholesterolemia have been identified as risk factors for IS.

Table 3. Genotype frequencies of HDAC9 gene polymorphisms in IS and control groups and their associations with IS

\begin{tabular}{|c|c|c|c|c|c|c|}
\hline Genotype & Case group & Control group & $x^{2}$ & $p$-value & OR & $95 \% \mathrm{Cl}$ \\
\hline \multicolumn{7}{|l|}{ rs723296 } \\
\hline $\mathrm{TT}$ & 130 (83.87) & 118 (92.19) & Reference & Reference & & \\
\hline TC & 25 (16.13) & $10(7.81)$ & 4.474 & 0.034 & 2.269 & $1.046-4.923$ \\
\hline $\mathrm{CC}$ & $0(0.00)$ & $0(0.00)$ & & & & \\
\hline $\mathrm{T}$ & 285 (91.94) & 246 (96.09) & Reference & Reference & & \\
\hline C & 25 (8.04) & 10 (3.91) & 4.179 & 0.041 & 2.158 & $1.016-4.582$ \\
\hline \multicolumn{7}{|l|}{ rs957960 } \\
\hline $\mathrm{AA}$ & $37(23.87)$ & $39(30.47)$ & Reference & Reference & & \\
\hline$A C$ & 74 (47.74) & $58(45.31)$ & 1.054 & 0.304 & 1.115 & $0.904-1.375$ \\
\hline CC & $44(28.39)$ & $31(24.22)$ & 1.012 & 0.314 & 1.187 & $0.847-1.662$ \\
\hline $\mathrm{AA} / \mathrm{AC}+\mathrm{CC}$ & & & 1.319 & 0.251 & 1.088 & $0.940-1.259$ \\
\hline$C C / A A+A C$ & & & 0.276 & 0.599 & 1.114 & $0.744-1.666$ \\
\hline A & 148 (47.74) & 136 (53.13) & Reference & Reference & & \\
\hline$C$ & $162(52.26)$ & $120(46.87)$ & 1.096 & 0.295 & 1.095 & $0.923-1.298$ \\
\hline
\end{tabular}

Values are presented as number (\%). IS : ischemic stroke, OR : odds ratio, $\mathrm{Cl}$ : confidence interval 
Moreover, genetic factors have been found to be particularly involved in the pathogenesis of IS, but certain associations between genetic factors and IS are replicated ${ }^{19)}$. HDAC9 polymorphisms affect lipid homeostasis, cholesterol efflux, platelet activation, and genes involved in inflammatory responses. Several studies have shown that genetic variation in 7 p21.1 chromosome is closely linked to IS caused by arterial disease $^{27)}$. The HDAC9 gene encodes proteins responsible for deacetylation of histones and in turn regulating the chromatin structure and gene transcription. Several studies have replicated that HDAC9 gene polymorphism is linked to susceptibility of IS. By way of Markus Carotid Artery Ultrasound (MCAO) found that a genetic variant of HDAC9 stimulates carotid plaque formation and increases the risk of stroke, and through isotope labeling found that the HDAC9 gene is highly expressed in the endothelial cells of blood vessels in the brain and myocardium ${ }^{29)}$. The MCAO results indicated that HDAC9 expression was found to be significantly increased in a rat model of cerebral ischemia reperfusion injury ${ }^{7)}$. Studies have shown that the HDAC9 SNP allele of rs 3757720 is associated with coronary heart disease, which is a marker of atherosclerosis $^{2}$. In the mice knockout HDAC9 gene, the extent of atherosclerosis lesions was decreased significantly ${ }^{4}$. This indicates that the absence of HDAC9 expression can result in the downregulation of inflammation-related genes and upregulation of lipid-related genes ${ }^{5,13)}$. As per several other related studies, the HDAC9 gene variant is associated with the risk of stroke, but results may vary with different genes.

A study conducted on 262 Chinese Han stroke patients, investigated the loci rs2389995 (A/G), rs2240419 (C/T), and rs1984041 (T/C) of the HDAC9 gene. No polymorphism of rs1984041 (C/T) locus was found in the Chinese Han population; the alleles A and T of genes rs2389995, rs2240419 were, respectively, found to increase the risk of stroke ${ }^{18)}$. In a casecontrol study performed on 279 Han stroke and 984 healthy population from the Second Military Medical University of Shanghai, alleles A and T of rs2389995 and rs2240419 were, respectively, observed to have no correlation with stroke risk. Subcomponent type was performed for stroke and alleles A and $\mathrm{T}$ of rs 2389995 , rs2240419 were, respectively observed to increase the risk of macrovascular stroke ${ }^{6}$. Liu et al. ${ }^{12)}$ conducted a study on SNP sites which included 45 Han Chinese patients of Beijing, and found that the binding of the HDAC9 gene rs2526630 to micro-RNA might be related to the inci- dence of atherosclerotic IS. From this it can be deduced that HDAC9 may contain risk susceptibility loci for stroke, and polymorphism of HDAC9 gene has racial differences among various ethnic populations.

In this study, TaqMan-PCR was performed to analyze 155 IS patients and 128 healthy Han individuals residing in Dali, and the correlation between rs1060499865, rs723296, and rs957960 polymorphisms of HDAC9 and stroke was confirmed. The rs1060499865 gene was located on Chromosome:7:18793416, genic downstream transcript variant, synonyms variant, and coding sequence variant. The rs723296 gene was located on Chromosome:7:18389685, intron variant, and genic upstream transcript variant. The rs957960 gene was located on Chromosome:7:18837785, intron variant, and genic downstream transcript variant. In this study, no polymorphism of the rs1060499865 gene was observed; both rs723296 and rs957960 appeared to be gene mutants. There was no homozygous CC at the rs723296 locus, hence, only select alleles $(\mathrm{C} / \mathrm{T})$ and additive (TC/TT) genetic models were analyzed. In the $\mathrm{C} / \mathrm{T}$ allele model ( $p=0.041$; OR, 2.158; 95\% CI, 1.0164.582), showed in rs723296 sites in patients with $\mathrm{C}$ allele were proved to be 2.158 -folds more IS risk associated than that of $\mathrm{T}$ allele. In additive genetic model TC/TT ( $p=0.034$; OR, 2.269; $95 \%$ CI, 1.046-4.923), illustrated in rs723296 sites in patients with TC gene was found to be 2.269 -folds more IS risk associated than that of $\mathrm{T}$ allele. The results indicated that the rs723296 locus was associated with the risk of stroke in individuals carrying the $\mathrm{C}$ allele and $\mathrm{CT}$ genotype that was higher than that in individuals carrying the T allele and TT genotype, which may be attributed to rs723296 polymorphisms that alter the acetylation-deacetylation balance and increase the risk of stroke. At the rs957960 site, in alleles, additive, dominant, or recessive models, gene mutations were not found to be associated with stroke, indicating no significant association between rs957960 polymorphism and the incidence of stroke in patients.

This study had some limitations : 1) our sample was selected from the Han Chinese population of Dali city, which renders the study inapplicable to other ethnicities; 2) regional disparity resulted in the possible inconsistencies in the role of the same SNP locus in case of the same disease occurring in different ethnic groups and different diseases occurring in the same ethnic group; and 3) inclusion and exclusion criteria of IS vary for different studies. 
Altogether, the results of this study demonstrate that polymorphism of the HDAC9 gene locus rs723296 increases the risk of IS in Han populations of Dali. Nevertheless, there was no polymorphism in locus rs1060499865; rs957960 polymorphism was not found to be significantly associated with the IS patients from the Dali region. Larger sample sizes need to be investigated for the analysis of the correlation between HDAC9 gene polymorphism and IS susceptibility in different regions and ethnic groups; moreover, the mechanism of gene polymorphism in IS pathogenesis needs to be further studied.

\section{CONCLUSION}

To conclude, the polymorphism of the gene HDAC9, rs723296 locus increases the risk of IS. TC genotype was observed to be associated significantly with the risk of IS in Han population of Dali. This finding of the study provides new insight for future explorations regarding IS pathogenesis.

\section{CONFLICTS OF INTEREST}

No potential conflict of interest relevant to this article was reported.

\section{INFORMED CONSENT}

Informed consent was obtained from all individual participants included in this study.

\section{AUTHOR CONTRIBUTIONS}

\author{
Conceptualization : XY \\ Data curation : XY \\ Formal analysis : DL, RM \\ Funding acquisition : GW, XY \\ Methodology : YZ, HX, DL \\ Project administration : GW \\ Visualization : $\mathrm{YZ}$ \\ Writing - original draft : XY \\ Writing - review \& editing : GW
}

\section{ORCID}

$$
\begin{array}{ll}
\text { Xitong Yang } & \text { https://orcid.org/0000-0002-6203-3313 } \\
\text { Hongyang Xu } & \text { https://orcid.org/0000-0001-8793-0849 } \\
\text { Dan Liu } & \text { https://orcid.org/0000-0001-9918-5436 } \\
\text { Rong Ma } & \text { https://orcid.org/0000-0002-1866-955X } \\
\text { Yuanyuan Zhang } & \text { https://orcid.org/0000-0002-5263-4895 } \\
\text { Guangming Wang } & \text { https://orcid.org/0000-0002-0220-1493 }
\end{array}
$$

\section{- Acknowledgements}

This study was supported by the National Natural Science Foundation of China (No. 81360206); Reserve Talents of Academic and TechnicalLeaders of Young and Middle-aged People in Yunnan Province (No. 2014HB025); Scientific research fund project of Yunnan education department (2019J0775); and Colleges joint specific project in Yunnan Province (2019FH001-(020)).

\section{References}

1. Baird AE : Genetics and genomics of stroke: novel approaches. J Am Coll Cardiol 56 : 245-253, 2010

2. Cao Q, Rong S, Repa JJ, Clair RS, Parks JS, Mishra N : Histone deacetylase 9 represses cholesterol efflux and alternatively activated macrophages in atherosclerosis development. Arterioscler Thromb Vasc Biol 34 : 1871-1879, 2014

3. Chinese Society of Neurology : The diagnosis of cerebrovascular diseases. CHN of J Neurol $29: 379-380,1996$

4. Ferronato S, Mombello A, Posenato I, Candiani P, Scuro A, Setacci C, et al. : Expression of circulating miR-17-92 cluster and HDAC9 gene in atherosclerotic patients with unstable and stable carotid plaques. Genet Test Mol Biomarkers 21 : 402-405, 2017

5. Han X, Han X, Wang Z, Shen J, Dong Q : HDAC9 regulates ox-LDLinduced endothelial cell apoptosis by participating in inflammatory reactions. Front Biosci (Landmark Ed) 21 : 907-917, 2016

6. Han Y, Sun W, Wang L, Tao S, Tian L, Hao Y, et al. : HDAC9 gene is associated with stroke risk in a Chinese population. Exp Biol Med (Maywood) $238: 842-847,2013$

7. He $\mathrm{M}$ : The role and mechanism of acetylation epigenetic regulation molecule HDAC4/5 in ischemic brain injury. Jinan : Shandong University, 2012

8. International Stroke Genetics Consortium (ISGC); Wellcome Trust Case Control Consortium 2 (WTCCC2), Bellenguez C, Bevan S, Gschwendtner A, Spencer CC, et al. : Genome-wide association study identifies a variant in HDAC9 associated with large vessel ischemic stroke. Nat Genet 44 : 328-333, 2012 
9. Kalkonde YV, Sahane V, Deshmukh MD, Nila S, Mandava P, Bang A : High prevalence of stroke in Rural Gadchiroli, India: a community-based Study. Neuroepidemiology 46 : 235-239, 2016

10. Kaluza D, Kroll J, Gesierich S, Manavski Y, Boeckel JN, Doebele C, et al. : Histone deacetylase 9 promotes angiogenesis by targeting the antiangiogenic microRNA-17-92 cluster in endothelial cells. Arterioscler Thromb Vasc Biol 33 : 533-543, 2013

11. Lahm A, Paolini C, Pallaoro M, Nardi MC, Jones P, Neddermann P, et al. : Unraveling the hidden catalytic activity of vertebrate class lla histone deacetylases. Proc Natl Acad Sci U S A 104 : 17335-17340, 2007

12. Liu WL, Lin YJ, Tao J, Peng J, Li GP, Chen LD, et al. : Study on the correlation between HDAC9 gene SNPs and large vessel atherosclerosisinduced ischemic stroke. Chin J Stroke 11 : 269-276, 2016

13. Lu S, Li H, Li K, Fan XD : HDAC9 promotes brain ischemic injury by provoking $\left.\right|_{k B} \alpha / N F-k B$ and MAPKs signaling pathways. Biochem Biophys Res Commun 503 : 1322-1329, 2018

14. Markus HS, Mäkelä KM, Bevan S, Raitoharju E, Oksala N, Bis JC, et al. : Evidence HDAC9 genetic variant associated with ischemic stroke increases risk via promoting carotid atherosclerosis. Stroke 44 : 12201225, 2013

15. Musunuru K, Hickey KT, Al-Khatib SM, Delles C, Fornage M, Fox CS, et al. : Basic concepts and potential applications of genetics and genomics for cardiovascular and stroke clinicians: a scientific statement from the American Heart Association. Circ Cardiovasc Genet 8 : 216-242, 2015

16. Petrie K, Guidez F, Howell L, Healy L, Waxman S, Greaves M, et al. : The histone deacetylase 9 gene encodes multiple protein isoforms. J Biol Chem 278 : 16059-16072, 2003

17. Prestel M, Prell-Schicker C, Webb T, Malik R, Lindner B, Ziesch N, et al. : The atherosclerosis risk variant rs2107595 mediates allele-specific transcriptional regulation of HDAC9 via E2F3 and Rb1. Stroke 50 : 26512660, 2019

18. Qingxu G, Yan Z, Jiannan X, Yunlong L : Association between the gene polymorphisms of HDAC9 and the risk of atherosclerosis and ischemic stroke. Pathol Oncol Res 22 : 103-107, 2016

19. Qingxu G, Yan Z, Jiannan $X$, Yunlong $L$ : Association between the gene polymorphisms of HDAC9 and the risk of atherosclerosis and ischemic stroke. Pathol Oncol Res 22 : 103-107, 2016
20. Smith JD : New role for histone deacetylase 9 in atherosclerosis and inflammation. Arterioscler Thromb Vasc Biol 34 : 1798-1799, 2014

21. Traylor M, Farrall M, Holliday EG, Sudlow C, Hopewell JC, Cheng YC, et al. : Genetic risk factors for ischaemic stroke and its subtypes (the METASTROKE Collaboration): a meta-analysis of genome-wide association studies. Lancet Neurol 11 : 951-962, 2012

22. Venketasubramanian N, Yoon BW, Pandian J, Navarro JC : Stroke epidemiology in South, East, and South-East Asia: a review. J Stroke 19 : 286-294, 2017

23. Wang M, Gu M, Li Z, Sun B, Cheng X, Dai Z, et al. : HDAC9 polymorphisms predict susceptibility, severity, and short-term outcome of large artery atherosclerotic stroke in Chinese population. J Mol Neurosci 67 : 165-171, 2019

24. Wang X, Sun Z, Zhang Y, Tian X, Li Q, Luo J : Impact of the PDE4D gene polymorphism and additional SNP-SNP and gene-smoking interaction on ischemic stroke risk in Chinese Han population. Neurol Res 39 : 351356, 2017

25. Wang XB, Han YD, Sabina S, Cui NH, Zhang S, Liu ZJ, et al. : HDAC9 variant Rs2107595 modifies susceptibility to coronary artery disease and the severity of coronary atherosclerosis in a Chinese Han population. PLoS One 11 : e0160449, 2016

26. Xu YL, Hu YY, Li JW, Zhou L, Li L, Niu YM : Aldehyde dehydrogenase 2 rs671G > A polymorphism and ischemic stroke risk in Chinese population: a meta-analysis. Neuropsychiatr Dis Treat 15 : 1015-1029, 2019

27. Yeo BPH, Foong LC, Tam SM, Lee V, Hwang SS : Gene isolation using degenerate primers targeting protein motif: a laboratory exercise. Biochem Mol Biol Educ 46 : 47-53, 2018

28. Zhou M, Wang H, Zhu J, Chen W, Wang L, Liu S, et al. : Cause-specific mortality for 240 causes in China during 1990-2013: a systematic subnational analysis for the Global Burden of Disease Study 2013. Lancet $387: 251-272,2016$

29. Zhu QB, Wu GM, Li GM, et al. : Study on the relationship between single nucleotides polymorphism rs2240419 of HDAC9 gene and the susceptibility to ischemic stroke and TCM syndromes in Guangdong Han population. Chinese Journal for Clinicians 47 : 35-40, 2019

30. Zhu R, Liu X, He Z, Li Q : MiR-146a and miR-196a2 polymorphisms in patients with ischemic stroke in the northern Chinese Han population. Neurochem Res 39 : 1709-1716, 2014 